\title{
Enseñanza virtual de la investigación de operaciones durante la COVID-19. Un análisis desde la práctica docente universitaria
}

\author{
Virtual teaching of operations investigation during COVID-19. An analysis from the university teaching practices
}

Ensino virtual de investigação operacional durante o COVID-19. Uma análise a partir da prática docente universitária

Juan Carlos Muyulema-Allaica
jmuyulema@pucesm.edu.ec
https://orcid.org/0000-0002-9663-8935

José Luis Gavidia-García ${ }^{3}$

jl.gavidia@uta.edu.ec

https://orcid.org/0000-0002-4077-9288

\author{
Roberto Bernardo Usca-Veloz ${ }^{2}$ \\ rusca@ueb.edu.ec \\ https://orcid.org/0000-0001-6600-052X
}

\author{
Paola Martina Pucha-Medina ${ }^{4}$ \\ pumepm@hotmail.com \\ https://orcid.org/0000-0002-4712-7661
}

${ }^{1,4}$ Pontificia Universidad Católica del Ecuador Sede Manabí, Portoviejo, Ecuador

${ }^{2}$ Universidad Estatal de Bolívar, Guaranda, Ecuador

${ }^{3}$ Universidad Técnica de Ambato, Ambato, Ecuador

${ }^{4}$ Grupo Consultor Empresarial CAAPTES, Riobamba, Ecuador

Recibido 1 de abril 2021 | Arbitrado y aceptado 27 de mayo 2021 | Publicado en 01 julio 2021

\section{ABSTRACT}

El objetivo del presente estudio fue recoger las reflexiones de la enseñanza virtual de la investigación de operaciones (IO) durante la COVID-19, partiendo de análisis desde la práctica docente universitaria. El método se fundamentó en un ciclo de investigaciónacción. El grupo de estudio estuvo conformado por una muestra de 30 docentes universitarios que dictan la asignatura de IO, estratificados en las provincias ecuatorianas de Bolívar, Chimborazo, Manabí, y Tungurahua. Se aplicó dos técnicas de recolección de datos, utilizando la plataforma Zoom y Google Forms. El instrumento fue evaluado por profesores investigadores. Los resultados evidencian que el $93.33 \%$ de los estudiantes universitarios soportan problemas en su proceso formativo durante la crisis sanitaria. En conclusión, el estudio, reveló profundas limitaciones de carácter organizativo, administrativo y pedagógico en la implementación de entornos virtuales de aprendizaje, y demostró, la necesidad del establecimiento de nuevos modelos de enseñanza virtual y de sistemas híbridos (presenciales y a distancia) para la docencia.

Key words:: Crisis sanitaria; docencia; educación superior; estudiantes; enseñanza virtual

\section{RESUMEN}

The objective of this study was to collect the reflections of the virtual teaching of operations research (OR) during COVID-19, based on analysis from the university teaching practice. The method was based on an action-research cycle. The study group was made up of a sample of 30 university teachers who teach the OR subject, stratified in the Ecuadorian provinces of Bolívar, Chimborazo, Manabí, and Tungurahua. Two data collection techniques were applied, using the Zoom platform and Google Forms. The instrument was evaluated by research professors. The results show that $93.33 \%$ of university students endure problems in their training process during the health crisis. In conclusion, the study revealed profound limitations of an organizational, administrative and pedagogical nature in the implementation of virtual learning environments, and demonstrated the need for the establishment of new virtual teaching models and hybrid systems (face-to-face and distance) for teaching.

Palabras clave: Health crisis; teaching; higher education; students; virtual teaching

\section{RESUMO}

O objetivo deste estudo foi coletar as reflexões do ensino virtual de investigação de operações (IO) durante o COVID-19, a partir da análise da prática docente universitária. $\mathrm{O}$ método foi baseado em um ciclo de ação de investigação. O grupo de estudo foi constituído por uma amostra de 30 professores universitários que lecionam a disciplina de OI, estratificados nas províncias equatorianas de Bolívar, Chimborazo, Manabí e Tungurahua. Duas técnicas de coleta de dados foram aplicadas, utilizando a plataforma Zoom e Formulários Google. $\mathrm{O}$ instrumento foi avaliado por professores pesquisadores. Os resultados mostram que $93,33 \%$ dos universitários enfrentam problemas em seu processo formativo durante a crise de saúde. Concluindo, o estudo revelou profundas limitações de ordem organizacional, administrativa e pedagógica na implementação de ambientes virtuais de aprendizagem, e demonstrou a necessidade de implantação de novos modelos de ensino virtual e sistemas híbridos (presencial e a distância) de ensino.

Palavras-chave: Crise de saúde; ensino; educação superior; alunos; ensino virtual 


\section{INTRODUCCIÓN}

Desde sus inicios, la humanidad ha tenido que transitar por cambios abruptos y fortuitos que han sido causa del replanteamiento de sus modelos de vida (Issa, et al., 2021). El contexto actual, marcado por la existencia de una nueva crisis sanitaria de carácter mundial producida por el coronavirus SARS-COV-2 (COVID-19) que inició en Wuhan, China en el año 2020, ha alterado en muchas formas la vida cotidiana de los ecuatorianos del siglo XXI (López, et al., 2021). Uno de los sectores más ampliamente afectados es, sin duda, la educación universitaria (Coronel, 2021; Ordorika, 2020). Así, la actividad docente universitaria presencial fue totalmente suspendida durante los momentos de mayor emergencia sanitaria, período comprendido entre marzo-junio de 2020 (Usca-Veloz, et al., 2021). De esta forma, las Instituciones de Educación Superior (IES) tuvieron que replanificar toda la docencia adaptándola a una modalidad de enseñanza virtual, online y a distancia para garantizar el aprendizaje de los estudiantes universitarios de forma segura (Castillo y Cabrera, 2021). Tras este período, la cierta recuperación de los indicadores sanitarios permitió retomar ciertas actividades educativas de manera presencial (Caicedo y Ruiz, 2021). Sin embargo, en la mayoría de las IES, mantuvieron como presenciales las actividades pedagógicas estrictamente necesarias, como las que implicaban prácticas de laboratorio, manteniendo, por tanto, el aprendizaje online en la mayoría de los cursos o asignaturas combinadas con enseñanzas sincrónicas y asincrónicas.

En este contexto, tomaron una gran relevancia las diferentes plataformas digitales de aprendizaje disponibles, que venían utilizando en el ámbito universitario antes de la crisis sanitaria en algunas fases del desarrollo docente, generalmente presencial (Gorozidis, et al., 2020). Sin embargo, cabe destacar que, en su mayoría, y principalmente en las carreras de disciplinas científicas, esta herramienta virtual se utilizaba como un medio complementario a la enseñanza presencial con el objetivo de entregar recursos de aprendizaje extra a los alumnos, tales como el syllabus, material de estudio e instrumentos de autoevaluación, para facilitarles la preparación de la asignatura, mientras que el principal sistema de enseñanza eran las clases magistrales presenciales (Digión y Álvarez, 2021).

Este caso, la disciplina científica sobre investigación de operaciones (IO) caracterizada por la aplicación teórica, métodos analíticos avanzados y técnicas especiales, que tienen como finalidad la solucióndeproblemasenlaplaneación, organización, integración, dirección y el control que se puedan producir en los diversos sistemas que existen tanto en la naturaleza como en aquellos creados por los seres humanos (López, et al., 2020; Salazar, 2021). Debido a su énfasis en la interacción humanotecnología y formal a su enfoque en aplicaciones prácticas el uso del e-learning o educación a distancia a través de medios tecnológicos como herramienta docente principal era, antes de la crisis sanitaria, bastante escaso e infrecuente (Usca-Veloz, et al., 2021). En este sentido, el paso a una docencia completamente online a través de estas plataformas que facilitan la teleformación en tiempos de crisis conjeturó un gran reto para las IES, puesto que tuvieron que adaptar y reforzar todos los recursos online disponibles para evitar su colapso debido a su progresivo uso y garantizar sistemáticamente el acceso de todos los estudiantes universitarios a estas plataformas (Vera, 2021). En estos escenarios, en los cuales interactúan, por un lado, los docentes, que tuvieron que replanificar completamente su método de enseñanza; y, por el otro, los alumnos, debieron adaptarse presurosamente a un nuevo régimen de aprendizaje en un entorno que, en algunos casos, no era adecuado para ello.

Por lo cual, hoy en día cobra relevancia indagar y conocer la mirada de los docentes universitarios de carreras profesionales en ciencias duras, respecto a 
su percepción del proceso de implementación de una educación online durante la contingencia sanitaria por COVID-19, particularmente en asignaturas de carácter práctico, como la IO cuyo lugar natural de trabajo es el laboratorio, bajo un método de enseñanza-aprendizaje presencial mediante clases magistrales por su nivel de complejidad en los desarrollos de técnicas matemáticas (algoritmos) que buscan soluciones a problemas ante una serie de restricciones. A razón de esto, el objetivo de esta investigación es recoger las reflexiones de la enseñanza virtual de la IO durante la COVID-19, partiendo de análisis desde la práctica docente universitaria. El estudio se fundamentó en un ciclo de investigación-acción. El grupo de estudio estuvo conformado por una muestra de 30 profesores universitarios que dictan la asignatura de IO, estratificados en cuatro importantes provincias ecuatorianas como son: Bolívar, Chimborazo, Manabí, y Tungurahua.

\section{MÉTODO}

La metodología del estudio aplicada se fundamentó en un ciclo de investigación-acción, constituidos por las siguientes fases: planificar, actuar, observar y reflexionar. El grupo de estudio estuvo conformado por profesores universitarios que dictan la asignatura de IO, y que trabaja habitualmente con esta metodología, uniendo de forma sistemática la innovación e investigación educativa. Las técnicas de recolección de datos se establecieron en dos:

- Dos videoconferencias específicas sobre este tema, en las que todos los participantes de forma espontánea comentan sus propias experiencias, utilizando la plataforma de video y audio conferencia online Zoom;

- Aplicación de un cuestionario semiestructurado elaborado ad hoc, diseñado con siete (8) ítems básicos cerrados y catorce (16) preguntas abiertas. $\mathrm{El}$ instrumento fue evaluado por profesores investigadores adscritos a la Pontificia Universidad Católica del Ecuador Sede Manabí (PUCEM) y una vez que fueron hechas las correcciones sugeridas, se aplicó el instrumento usando un software de administración de encuestas que se incluye como parte del paquete gratuito de editores de documentos, Google Forms.

La muestra objeto de estudio estuvo compuesta por 30 profesores universitarios, que ejercen su función docente en las provincias ecuatorianas de Bolívar, Chimborazo, Manabí, y Tungurahua. En la Tabla 1, se presenta los principales datos descriptivos de la muestra de estudio.

Tabla 1. Principales datos descriptivos de la muestra objeto de estudio.

\begin{tabular}{ccccc}
\hline $\mathbf{N = 3 0}$ & $\begin{array}{c}\text { Datos descriptivos de } \\
\text { la muestra }\end{array}$ & Opciones & Número & Porcentaje (\%) \\
\hline \multirow{2}{*}{ Variables Descriptivas } & Provincia & Bolívar & 7 & 23.33 \\
& & Chimborazo & 11 & 36.67 \\
& & Manabí & 3 & 10.00 \\
& \multirow{3}{*}{ Entorno } & Tungurahua & 9 & 30.00 \\
& & Urbano & 28 & 93.33 \\
& & Rural & 2 & 6.67 \\
\hline
\end{tabular}




\begin{tabular}{|c|c|c|c|c|}
\hline $\mathbf{N}=\mathbf{3 0}$ & $\begin{array}{c}\text { Datos descriptivos de } \\
\text { la muestra }\end{array}$ & Opciones & Número & Porcentaje (\%) \\
\hline \multirow[t]{9}{*}{ Variables Descriptivas } & \multirow{4}{*}{$\begin{array}{l}\text { Estratos del nivel } \\
\text { sociocultural del } \\
\quad \text { alumnado }\end{array}$} & Alto & 2 & 6.67 \\
\hline & & Medio & 17 & 56.67 \\
\hline & & Medio-bajo & 7 & 23.33 \\
\hline & & Bajo & 4 & 13.33 \\
\hline & \multirow[t]{2}{*}{ Titularidad } & Pública & 25 & 83.33 \\
\hline & & Privada & 5 & 16.67 \\
\hline & \multirow[t]{3}{*}{ Nivel educativo } & Quinto semestre & 12 & 40.00 \\
\hline & & Sexto semestre & 9 & 30.00 \\
\hline & & Séptimo semestre & 9 & 30.00 \\
\hline
\end{tabular}

Fuente: Base de datos de la investigación.

El análisis de los datos se ejecutó utilizando un proceso de categorización previo, por medio del cual se buscó reducir la información de la investigación con el fin de expresarla y describirla de modo conceptual, de tal forma que respondan a una estructura sistemática, inteligible para otras personas, y por lo tanto significativa. En la Tabla 2 se muestra la información agrupada en torno a un sistema de tres (3) categorías principales y trece (13) subcategorías.

Tabla 2. Sistema de reducción en categorías y subcategoría.

\begin{tabular}{lll}
\hline No. & Categorías & Subcategorías \\
\hline
\end{tabular}

1 Cómo vive el docente de la asignatura de

1.1. Entendimiento del ambiente del alumnado y el de sus familias. IO esta situación

1.2. Los docentes con alta carga de trabajo y los centros educativos exigiendo accesos a la educación por todos los medios.

1.3. La brecha digital y el acceso limitado a las tecnologías.

1.4. Nativos e inmigrantes digitales y alumnos desconectados.

1.5. Utilidad de los esfuerzos docentes para generar aprendizaje.

1.6. El papel del docente en la educación y la vida del alumnado.

1.7. Docentes de IO, y sus familias 


\begin{tabular}{llc}
\hline No. & Categorías & Subcategorías \\
\hline
\end{tabular}

2 Cómo vive el docente de la asignatura de IO esta situación
2.1. Contenidos impartidos

2.2. Aplicaciones informáticas utilizadas

2.3. Respuesta de las autoridades universitarias

2.4. Niveles de respuesta de los estudiantes universitarios.
3 Cómo plantar evaluaciones y establecer calificaciones del curso de IO, en un entorno de enseñanza online.
3.1. Métodos de evaluación durante el confinamiento.

3.2. Métodos de calificación del curso de IO.

Fuente: Técnica aplicada al grupo objeto de estudio.

RESULTADOS

Los resultados del proceso investigativo se han organizado sistemáticamente en tres (3) grandes apartados. El primero, se refirió al cómo vive el docente universitario que dicta la materia de IO. Se procedió a expresar diversos dilemas y ciertas reflexiones. El segundo, se representó lo que está haciendo el docente universitario que dicta la materia de IO, ante la situación de confinamiento social por la Covid-19 y los procesos de enseñanza online. Finalmente, en el tercer apartado se expresó los resultados concernientes a cómo plantar evaluaciones y establecer calificaciones del curso de IO, en un entorno de enseñanza online.

\section{Cómo vive el docente universitario que dicta la asignatura de IO}

En este apartado se presenta los resultados que hicieron referencia a cómo vive el docente universitario que dicta la materia de IO, en el entorno de confinamiento causado por el confinamiento por la COVID-19.

\section{A. Entendimiento del ambiente del alumnado $y$ el de sus familias}

Los resultados evidencian que, en todo este periodo, el docente universitario del grupo objeto de estudio se ha enfrentado a diversas realidades familiares. Familias pasando por situaciones complicadas en referencia a la salud (enfermedad por la Covid-19 y/o la pérdida de un familiar); familias que han perdido su puesto de trabajo $y$ por ende transitan en verdaderos problemas económicos para afrontar estos meses y los que faltan de pandemia; familias con pocos recursos y deficientes conocimientos tecnológicos; familias que se ven en la necesidad de trabajar desde casa y cuidar al mismo tiempo de los menores a su cargo; o a su vez familias privilegiada, puesto que no sufren ninguna de las dificultades antes expresadas y además pueden sin ningún problema dedicar tiempo a sus familias y a sus aprendizajes escolares. Más la situación generalizada hacia la adopción de algunas sencillas medidas de precaución al salir de casa.

Estas situaciones tan dinámicas y diferentes, genera en el académico dudas e inseguridades sobre cómo organizar los compendios docentes. ¿Qué contenidos priorizar?, ¿Qué medios utilizar para llegar a todos?, ¿Qué estrategia metodológica de enseñanza aprendizaje utilizar?, ¿Cuáles son las herramientas tecnológicas más adecuadas?, ¿Cómo evaluar al estudiante universitario?, ¿Es proporcionado lo que se está requiriendo; es mucho, poco, adecuado?

Por otra parte, los resultados expresan la diversidad de instituciones de educación superior (IES) y contextos socio-educativos (Universidades 
Publicas y Privadas). Las universidades que habían iniciado una transición a la digitalización antes de la pandemia y contaban con una infraestructura tecnológica ya tenían cierta experiencia en el desarrollo de una cultura digital, con estudiantes y profesores más adaptados a mecanismos como trámites digitalizados y cursos presenciales dictados en un formato híbrido y con el contenido curricular en línea.

En este instante se cree trascendental exponer la importancia de la IO en la formación universitaria, puesto que esta permite la solución de problemas complejos a través del uso de múltiples disciplinas, por lo que es sumamente necesario conocer estos sistemas para tomar mejores decisiones y aumentar la productividad organizacional. En este sentido, la IO provee al estudiante de ciencias duras de una "caja de herramientas" que puede ser usada en una amplia gama de situaciones; por ejemplo: en producción ayuda a planificar la asignación de los recursos con el objeto de maximizar las utilidades o minimizar los costos, con el objeto de maximizar los beneficios de la misma con los recursos y restricciones que se tengan, en otras áreas administrativas con IO se puede diseñar un portafolios que maximice el rendimiento esperado para un cierto nivel de riesgo, también ayuda a planificar y controlar el inventario.

Teniendo presente, esta finalidad, y siendo conscientes de que las situaciones excepcionales requieren de medidas excepcionales y una flexibilidad también espacial. La flexibilidad debe permitir sacar la mayor potencialidad de los estudiantes universitarios, que, si estén en condiciones se seguir el ritmo y avanzar en sus procesos de aprendizaje, pero por otro lado se debe pensar en las condiciones familiares menos favorables. La labor docente en estos escenarios de pandemia va mucho más halla, puesto que debe ir enfocada a la búsqueda de un equilibrio psicológico y emocional del grupo estudiantil, sin obsesionarse sobre el ritmo de avance.

\section{B. Los docentes con alta carga de trabajo y los centros educativos}

Los docentes universitarios que dictan la materia de IO dialogan sobre su vivencia profesional en esta situación de pandemia tan repentina e inesperada, claramente la mayoría el $86.66 \%$ del grupo objeto de estudio diferencia claramente tres situaciones al inicio de la virtualidad por pandemia:

- Deficiente información sobre posibles recursos relacionados con las tecnologías de la información y la comunicación (TIC).

- Espacios para organizar la asignatura y falta de formación en recursos TIC.

- Falta de apoyo institucional para resolver esta situación.

Esta visión cambio con el paso del tiempo (segundo periodo ordinario), donde la mayor parte de los docentes universitarios expresan haberse acostumbrado a la rutina de trabajo y por ende agonizar y planificar de mejor manera los contenidos de la materia para un segundo periodo. Por otro lado, el $\mathbf{9 3 . 3 3 \%}$ de los académicos que dictan la materia de IO aprovecho el tiempo para acudir a formación por iniciativa propia, aunque adicionalmente el 70,00\% de los docentes exponen que han aprovechado los cursos online sobre TIC que ofertaron las autoridades educativas ante los entornos de pandemia.

Por otro lado, hay que tomar en cuenta que no todas las IES poseen estrategias de continuidad de la actividad docente y, en su ausencia, los contratos temporales pueden quedar inválidos (Caicedo y Ruiz, 2021). En la muestra estudiada la docencia universitaria con dedicación exclusiva no está generalizada y un $60.00 \%$ de los docentes tienen dedicación a tiempo parcial. Amas de este senario se debe considerar que, el impacto más evidente sobre los docentes está siendo la expectativa, cuando no exigencia, de la continuidad de la actividad docente 
bajo la modalidad virtual. Por lo menos en teoría, la educación virtual está presente en la mayoría de las grandes IES y es difícil encontrar una que no cuente con un campus virtual y, en su seno, de un aula virtual para cada asignatura, como extensión del aula física.

\section{La brecha digital y el acceso limitado a las tecnologías}

En la comentada situación de pandemia, y enseñanza online, una de las mayores preocupaciones que expresa la muestra estudiada de docentes universitarios que dictan la materia de IO, es cómo intentar superara la denominada "brecha digital y el acceso limitado a las tecnologías". Los docentes expresan que muchos estudiantes tienen dificultades para acceder a computadoras o no tienen conectividad a su disposición, para poder trabajar online los diferentes contenidos educativos, otros no cuentan con el apoyo suficiente por parte de su familia, o a su vez no poseen espacios adecuados, para seguir adelante con estos procesos de educación online; o simplemente las actuales condiciones los han obligado a dejar de estudiar y buscar una fuente de ingreso para apoyar a su familia, lo cual resulta en un incremento de la tasa de abandono de las aulas. Ante esta situación de educación online, todos estos factores hacen que la brecha digital y el acceso limitado a las tecnologías se proyecta a más situaciones.

La utilización de dispositivos móviles diferentes del ordenado (móviles inteligentes, o tabletas), según la muestra estudiada genera otro tipo de dudas y preocupaciones: ¿Cómo hacer llegar a los estudiantes universitarios los diferentes contenidos de la materia?, ¿Cómo afecta esto a la normativa de protección de datos? Bajo estos contextos, el $86.66 \%$ de los docentes universitarios que dictan la materia de IO, están de acuerdo con utilizar los recursos y aplicaciones oficiales que ofrecen las IES (correo electrónico institucional, plataformas online, aulas virtuales, entre otros), y así evitar toda la posible problemática relacionada con el uso de documentos, imágenes y protección de datos.

\section{Nativos e inmigrantes digitales y alumnos desconectados}

Los rápidos avances de la ciencia, la tecnología y la influencia de la globalización en la modernidad, los adelantos, la facilidad, la flexibilidad y la rapidez de la era digital hacen evidente el desarrollo de nuevas habilidades que influyen tanto en la vida social como académica de quien se encuentra en contacto directo con todos estos aspectos (Monterubbianesi, et al., 2021). Bajo este contexto, la denominación conceptual de (Buitrago, et al., 2020) establece una nueva nomenclatura, la cual designa a los profesores como "inmigrantes digitales" y a los estudiantes como "nativos digitales". Los inmigrantes son quienes se han adaptado al uso de los medios digitales usualmente ya en su edad adulta y los nativos quienes han crecido de la mano de la tecnología con habilidades innatas en el lenguaje del entorno digital (Salas, 2020). Por consiguiente, surgen dos perfiles diferentes, los cuales establecen una brecha respecto a las concepciones, los paradigmas, las visiones, el acceso, el uso y el manejo del lenguaje tecnológico.

Los nuevos retos en el proceso educativo plantean el uso de TIC de manera adecuada a través de estrategias metodológicas de enseñanza más modernas (Díaz, et al., 2021). Esto exige capacitación y formación continua para el desarrollo de habilidades y competencias tanto en estudiantes como en profesores, sin dejar de lado la ética, los valores y la construcción del conocimiento de forma colectiva (Gorozidis, et al., 2020).

Los docentes universitarios consultados expresan que impartir clases online supone sustentarse en una cultura de pedagogía virtual, puesto que consideran que no solo es un cambio de escenario del aula al hogar. La experiencia en 
entornos de confinamiento y cierre de IES ha demostrado que la competencia digital no se halla en un nivel adecuado de desarrollo en un porcentaje significativo de estudiantes, a pesar que mucho se ha discutido sobre ella y los recursos investidos para dicho fin. El 93.33\% de los académicos que dictan la materia de IO desde su experiencia expresan que desde el inicio de las actividades académicas online se ha evidenciado diversas particularidades que coexisten entre los estudiantes universitarios, supuestos "nativos digitales", pero que, a la hora de demostrar su conocimiento y destreza informática, a la mayoría, les resulta difícil seguir la cátedra por medio de entornos virtuales de aprendizaje en la educación a online.

Los datos demuestran que varias familias no tienen suficientes ordenadores para poder teletrabajar y estudiar en un escenario conformado por padres e hijos. Por otra parte, en entorno pandémico ha arrollado significativamente al grupo estudiantil más desfavorecido; los estudiantes desconectados. Este grupo de alumnos trasmitan por dos situaciones verdaderamente problemáticas, por un lado, se tiene el cierre las IES y por el otro su entorno sociocultural.

Por todo ello, el reto actual al que se enfrenta el docente universitario que dicta la materia de IO es cómo encontrar el equilibrio entre toda la diversidad de estudiantes, intentando garantizar la igualdad de oportunidades y el derecho a un aprendizaje significativo de alto nivel conceptual y emocional. En esta situación el 86.66\% del grupo objeto de estudio expresa que es trascendental trabajar más a fondo el proceso de digitalización iniciado en los entornos pandémicos y formar al estudiante universitario en estas competencias para un mejor uso y conocimiento de las mismas.

\section{E. Utilidad de los esfuerzos docentes para generar aprendizaje}

En este apartado, una de las preguntas que surgió por parte de los docentes universitarios indagados que dictan la materia de IO es: ¿Hasta qué punto lo que hacemos en la asignatura de IO es útil para la vida y práctica profesional de nuestros alumnos, especialmente en los entornos actuales de pandemia?

En condiciones habituales, se entiende que la importancia de la IO, posee diversas características:

- Para investigar los problemas utiliza el método científico de investigación.

- El proceso de investigación inicia con la observación de problemas y recolección de datos.

- El problema se presenta de forma cuantitativa, sólo de esta forma es posible su análisis y evaluación.

- El objetivo de este método es solucionar problemas organizacionales.

- Se encarga de encontrar la mejor alternativa para la solución del problema.

- Para que este método funcione es necesario realizar el trabajo en equipo, el cual debe estar formado por expertos.

Es cierto que la mayoría de los estudiantes y profesionistas, temen un poco a las matemáticas, incluso no las consideran tan importante en su formación profesional, sin embargo, es increíble la forma que, en las matemáticas, particularmente, la IO ayuda a realizar una administración de empresas más efectiva y exitosa. Y ahí radica la importancia de las matemáticas en todos los aspectos de vida. 
En la actualidad, el estado de confinamiento y posteriores mediadas de prevención nos obliga a desarrollar una labor docente online. Los extornos actuales de enseñanza-aprendizaje son muy complejos para todos los agentes educativos (docentes, estudiantes y familiares), por lo que es fundamental tener mucha flexibilidad, según la situación de cada estudiante.

La muestra de estudio, expresa que, como docentes de IO se busca desarrollar integralmente al estudiantado basado en estudios acerca del problema de planificación de las rutas de transporte (VRP, Vehicle Routing Problem), que han beneficiado es uno de los problemas de optimización combinatoria más importantes y ampliamente estudiado, con aplicaciones del mundo real, en la logística de distribución y del transporte. Esta contribución siempre ha estado implícita y los investigadores no han sido totalmente conscientes de las connotaciones beneficiosas de sus aportes. El problema VRP posee por objeto reducir al mínimo las distancias y la cantidad de vehículos asignados, se logra a la vez una disminución en el impacto ambiental, al reducir la cantidad de combustible y por resultado las emisiones de $\mathrm{CO}_{2}$ del transporte por carretera. Además, en tiempos de COVID-19, se debe tener una organización en el transporte óptima de recursos médicos, la cual puede influir en la toma de decisiones.

\section{F. El papel del docente en la educación y la vida del alumnado}

El título del apartado, nuevamente surgió por parte de los docentes universitarios indagados que dictan la materia de IO, una interrogante: ¿Cómo respondemos desde la IO a las diferentes situaciones, para garantizar una educación de calidad?

El $93.33 \%$ de la muestra de académicos universitarios que dictan la materia de IO desde su experiencia expresan que el reto para la IES ante la COVID-19 es conducir esta crisis a un aprendizaje significativo y constructivo en vez del debilitamiento del estudiante y en el peor de los casos a su deserción. Mientras se tenga la posibilidad de trabajar de modo online, por medio de un equipo informático, o mediante un folleto guía de estudios el docente es citado al cumplimiento de la misión de educar, de promover el pensamiento crítico y perfilar valores en la nueva ciudadanía a nivel global.

En este sentido, la postura del 100\% de los catedráticos de IO defiende la asignatura puesto que es un área fundamental en los actuales contextos pandémicos, puesto que la IO se aplica a problemas que se refieren a la conducción y coordinación de operaciones (o actividades) dentro de una organización. La naturaleza de la organización es esencialmente inmaterial y, de hecho, la IO se ha aplicado de manera extensa en áreas tan diversas como la manufactura, el transporte, la constitución, las telecomunicaciones, la planeación financiera, el cuidado de la salud, la milicia y los servicios públicos, por nombrar sólo unas cuantas. Así, la gama de aplicaciones es extraordinariamente amplia.

\section{G. Docentes de IO, y sus familias}

El 73.33\% de dos docentes de IO son padres o madres. En el 56.66\% de los casos los hijos e hijas se encuentran en etapas de educación obligatoria, por ente el entorno pandémico actual ha afectado doblemente: como docentes y como padres/madres de estudiantes. Así pues, incurriendo en ajuste de horarios o coincidencias en reuniones entre el grupo familiar, originando inconvenientes en el uso del ordenador $\mathrm{u}$ ordenadores con los que se cuenta o a su vez la recepción de información por los diferentes canales telemáticos, por ejemplo: organización del trabajo docente, avances del estudiantado (grupos de 60 a más estudiantes), o simplemente tareas e información de hijos o hijas.

Esta doble presión es más fuerte en hijos o hijas en edades cortas (infantil, primeros ciclos de 
primaria), puesto que son dependientes y se tiene que combinar actividades constantemente, a lo largo del día. Estas situaciones han comprometido los fines de semana del docente para ajustarse al requerimiento de las múltiples actividades.

\section{Cómo está resolviendo el entorno de confinamiento social y enseñanza online}

En este apartado, se buscó exponer tres aspectos fundamentales de la actuación docente durante el periodo de confinamiento social y los procesos de enseñanza online.

\section{A. Contenidos impartidos}

En este apartado se muestra los diferentes contenidos trabajados por los docentes universitarios indagados que dictan la materia de IO desde que comenzó el entorno pandémico.

Para el análisis, se ha tomado como referencia los bloques de contenidos correspondiente a la asignatura de IO, en dos periodos académicos, por un lado, el Ex-ante COVID-19 y, por otro lado, durante COVID-19, en estos escenarios el 93.33\% de académicos universitarios que dictan la asignatura de IO indicaron que los contenidos han variado, dando prioridad al trabajo autónomo.

Por ahora, los catedráticos exponen que no es posible determinar con certeza el impacto que tendrá la crisis en la implementación curricular en los distintos niveles de la enseñanza universitaria, pero se prevé una profundización de las diferencias en lo referente a los logros de aprendizaje, debido a las desigualdades educativas imperantes y a un acceso desigual a la cobertura curricular.

\section{B. Aplicaciones informáticas utilizadas}

Los datos obtenidos de la muestra de académicos universitarios que dictan la materia de IO desde su experiencia expresan que existe una seria deficiencia en varios hogares, que dificulta y en algunos casos obstaculiza el acceso a una educación no presencial, como la que exige la coyuntura actual. Y si se trata de estudiantes dentro de grupos vulnerables, con ingresos insuficientes para cubrir sus necesidades, víctimas de violencia o catástrofes, con familias sin empleo, infraestructura muy limitada, inexistencia de conexión a internet o estudiantes con alguna discapacidad, la situación educativa se hace más difícil e implica un mayor reto. En este contexto, la educación virtual se ha comenzado a impartir a través de:

- Plataformas de educación virtual: Moodle, SWAD (Social Workspace At a Distance), Chamilo, Google Classroom y otras.

- Aplicaciones de videoconferencias: Google meet, Zoom, Teams, Jitsi meet y otras.

- Aplicaciones de mensajería instantánea: WhatsApp, Messenger, Facebook y otras.

- Correo electrónico: Outlook, Gmail, Yahoo y otros.

- Señales de televisión: con el programa Aprendamos.

- Radioemisoras: con programas de aprendizaje.

Del amplio abanico de plataformas existentes que los docentes universitarios que dictan la materia de IO han utilizado, en mayor medida se encuentran las aulas virtuales Moodle y el correo electrónico corporativo, este último utilizado por un $46.66 \%$ de los docentes. También destacan otras aplicaciones con más del $40.00 \%$ de los docentes utilizan la herramienta Teams del paquete Office 365. Otra parte de los docentes del grupo de estudio ha optado por trabajar en la creación de blogs personales y páginas web como: Google Pages, Google Classroom, y canales de YouTube. Un número considerable de docentes (50\%) utiliza las demás herramientas combinadas con la App Zoom para videoconferencias. Un grupo reducido 
(16.66\%) de docentes usan redes sociales como WhatsApp o Instagram para comunicarse con los estudiantes.

Muchas de las aplicaciones descritas anteriormente son albergadas en aplicaciones de almacenamiento o nubes como One Drive del paquete Office 365, Drive de Google; o igualmente alojadas y presentadas en los blogs diseñados por los docentes, páginas web, sites, Classroom, y el Aula virtual.

\section{Respuesta de las autoridades universitarias}

Los sistemas educativos han tenido que responder urgentemente a una nueva situación: la imposible presencialidad en las IES y lo cierto es que en su mayoría no estaban preparadas para responder a ese reto (Ordorika, 2020). Así, los estudiantes y docentes se ven impactados por el cese temporal de actividades presenciales, readaptando sus actividades académicas a un modelo virtual, sin suficiente capacitación, apoyo y recursos (Digión y Álvarez, 2021).

La educación virtual permite mayor flexibilización en cuanto al manejo de los tiempos, espacios, distancias, y brinda mayores y mejores oportunidades de aprendizaje a las comunidades educativas (Coronel, 2021). Sin embargo, necesita condiciones de alistamiento digital, que implican la capacidad de conexión de estudiantes y docentes, dentro de otros rasgos (Moser, et al., 2021). Estas últimas condiciones, según Caicedo y Ruiz, (2021) generan desigualdades en el acceso a la educación virtual por la carencia de infraestructura y la falta de formación de los involucrados.

En líneas generales, el $86.66 \%$ de los docentes universitarios del grupo de trabajo expresaron que la actuación de los equipos directivos ha sido buena, puesto que la implementación de la enseñanza virtual representó un desafío para las autoridades universitarias, docentes y estudiantes, debido a ciertas consideraciones necesarias para su adecuada aplicación como: la disponibilidad de internet, un ambiente adecuado para estudiar en casa, el manejo de plataformas virtuales por parte de docentes y estudiantes, capacidad del docente para dictar cursos a distancia, un soporte académico tecnológico de la universidad, sílabos adaptados a una enseñanza virtual.

\section{Niveles de respuesta de los estudiantes universitarios}

Ante esta situación pandémica que está tocando a todos los eslabones que compones la comunidad educativa, en especial el estudiantado, se considera trascendental analizar la respuesta de éste, ante los bloques de contenidos correspondientes a la asignatura de IO, en dos periodos académicos, por un lado, el Ex-ante COVID-19 y, por otro lado, Durante COVID-19. Para dicho fin se tomó en cuenta dos factores: el nivel socio cultural y el tipo de IES (pública o privada).

En cuanto al nivel sociocultural del alumnado que concurrieron en los periodos analizados a los IES, son de nivel medio (56.67\%), seguido de un nivel medio-bajo (23.33\%), niveles bajo (13.33\%), y pocos niveles altos (6.67\%). Estos datos presentan resultados heterogéneos y la tendencia de los mismos no es clara. Sin embargo, se observa como las IES con estudiantado de nivel alto (uno público y otro privado), ejecutan un seguimiento del 100\% de las actividades docentes. En cambio, del nivel medio-bajo y bajo el seguimiento de los estudiantes es relativamente menor. Esta circunstancia según el 73.33\% de los docentes de asignatura de IO, se da porque por el entorno pandémico se procedió a agrupar un número considerable de estudiantes (en algunos casos más de 60 estudiantes) en un solo curso, ocasionando un alto nivel de trabajo docente. Otras variables que mencionan los docentes es la poca dedicación que le brindan al curso en las horas fuera de clase y malos hábitos de estudio. $\mathrm{Si}$ bien en el programa del curso se establece 
claramente la cantidad de horas mínimas de estudio independiente que deben dedicar (6 horas como mínimo), no llegan a cumplirlas.

\section{Cómo plantar evaluaciones y establecer calificaciones del curso de IO}

En este apartado se busca evidenciar el resultado de dos aspectos que se consideran fundamentales para docentes universitarios del grupo de trabajo, en consecuencia, surgió dos interrogantes: ¿Cómo plantear evaluaciones del curso de IO durante el confinamiento?, y ¿Qué método de calificación utilizar al finalizar el curso de IO?

\section{A. Métodos de evaluación durante el confinamiento}

Los docentes universitarios del grupo de trabajo expresaron que poseen muchos años de experiencia con sistemas y dinámicas de evaluación formativa universitaria, y diferenciado clara $y$ estratégicamente entre una evolución y calificación. En este sentido, con el fin de verbalizar el concepto, de forma conjunta se precisa que cuando se habla de evaluación, se refiere al proceso de evaluación formativa que se puede llevar a cabo a cada espacio hasta finalizar la asignatura. En cambio, en lo que se refiere a calificación de forma concreta, son los criterios a tener en cuenta para fijar la nota del estudiantado al final de la asignatura, así como el procedimiento a seguir para dicho cálculo.

En este sentido, los docentes universitarios del grupo de trabajo opinaron que un aspecto muy relevante es la evaluación y monitoreo de los aprendizajes, así como la retroalimentación, para conocer el progreso de las y los estudiantes y tomar las acciones pedagógicas pertinentes a fin de mejorarlo. Las actividades de educación a distancia han reafirmado la función formativa de la evaluación en las IES. La información sobre el aprendizaje individual de cada estudiante, a través de ejercicios de diagnóstico y de seguimiento, permite a las y los docentes proporcionar retroalimentación a sus estudiantes y modificar sus estrategias pedagógicas para que sean más efectivas. El desarrollo de instrumentos de evaluación formativa y de autoevaluación permite, además, fomentar los procesos de evaluación a cargo de las y los docentes universitarios en conjunto con sus estudiantes, para evaluar el avance de estos con respecto a las metas de aprendizaje propuestas.

\section{B. Métodos de calificación del curso de IO}

Los datos analizados muestran que el $70.00 \%$ de los docentes universitarios del grupo de trabajo presentaron inconvenientes con el tema de calificaciones desde el inicio del confinamiento, puesto que las entidades de control, ni las diferentes autoridades de los IES emitían indicaciones sobre el tema.

A pesar de que en los mementos actuales (principios de junio 2021) las autoridades de los IES ya han regulado el tema de la "Calificación", el $33.33 \%$ de los de los docentes universitarios del grupo de trabajo expresan tener ciertas dudas sobre como calificar al respecto. Por otro lado, el $66.66 \%$ de los participantes expresan tener pleno conocimiento a la hora de calificar, considerando que es imprescindible analizar la calidad de la enseñanza remota recibida y las dificultades personales y académicas que han enfrentado los estudiantes a fin de disponer de diagnósticos basados en evidencias que permitan a las autoridades universitarias diseñar escenarios educativos en el corto y medio plazo.

Se parte de la premisa, como afirman López, et al., (2021); Vera (2021); Coronel (2021) de que la educación a distancia implica la planificación y el diseño de experiencias de enseñanza y aprendizaje online. Sin embargo, la celeridad con la que las instituciones de educación superior tuvieron que adoptar la medida del cierre de las aulas no dejó margen de maniobra para realizar estas tareas, de 
ahí el concepto de enseñanza remota de emergencia (Portillo, et al., 2020). En este sentido, a pesar de haberse tomado decisiones importantes en materia educativa, como es el caso de la implementación masiva de la virtualidad en un tiempo relativamente corto; se revelan insuficiencias profundas en los métodos de calificación y, sobre todo, en las prácticas pedagógicas apoyadas en recursos tecnológicos poco conocidos por la mayoría de los docentes y estudiantes.

\section{Discusión}

A nivel global, una gran cantidad de estudiantes se han visto afectados de distintas maneras. La cancelación de clases presenciales y los estudios en línea, organizados con premura y preparación insuficiente, han generado grandes dificultades $y$ se han incrementado los procesos de exclusión y marginación (Awidi, et al., 2019). La UNESCO ha señalado que el mundo no estaba preparado para una disrupción educativa a semejante escala, en la que de la noche a la mañana escuelas y universidades del mundo cerraron sus puertas, apresurándose a desplegar soluciones de educación a distancia para asegurar la continuidad pedagógica (Saura, 2020). En este contexto global de emergencia, Ecuador no es una excepción, con apenas 1 de cada 2 hogares con servicio de Internet de banda ancha, y con ausencia de planes de contingencia para enfrentar el cambio del modelo presencial al modelo educativo a distancia, lo que ha impactado de manera inédita a todos los actores de la educación superior (UscaVeloz, et al., 2021).

Los resultados evidencian que, en todo el tiempo de confinamiento y en prácticas vigentes de salud y seguridad, el docente universitario del grupo objeto de estudio se ha enfrentado a diversas realidades familiares, laborales y sociales. En estos entornos surgen dudas e inseguridades sobre cómo organizar los compendios docentes y poder enfocar la IO en esta situación. ¿Qué contenidos priorizar?,
¿Qué medios utilizar para llegar a todos?, ¿Qué estrategia metodológica de enseñanza aprendizaje utilizar?, ¿Cuáles son las herramientas tecnológicas más adecuadas?, ¿Cómo evaluar al estudiante universitario?, ¿Es proporcionado lo que se está requiriendo; es mucho, poco, adecuado?

Las Tecnologías de la Información y Comunicación, comúnmente denominadas como TIC, son herramientas que representan un conjunto de tecnologías que tienen como denominador común el uso del código binario (bit) para representar y trabajar información de forma digital (Díaz, et al., 2021). Desde su aparición han provocado un cambio significativo en la organización productiva y social sobre las que se consolidan las bases de las llamadas Sociedades de la Información y en la vida de las personas comunes y corrientes (Kang y Jae, 2017). Ecuador está bastante estancado en lo que se refiere a TIC. En 2016, el país se ubicó en el 82 lugar en el mundo entre 139 países analizados, con un puntaje global de 3.9/7 en TIC, detrás de México, Uruguay, Argentina, Chile, Costa Rica, Brasil, Colombia y Venezuela, en ese orden, según indicadores de la Unión Internacional de Telecomunicaciones (ITU), con sede en Suiza (Usca-Veloz, et al., 2021). También existe una gran brecha digital dentro del país: puesto que solo el $40.40 \%$ de la población de Ecuador ha utilizado Internet en los últimos 12 meses. En el área urbana el $47.60 \%$ de la población ha utilizado internet, frente al $25.30 \%$ del área rural. Aún más preocupante, solo $4.0 \%$ de los residentes rurales cuenta con Internet en casa (García, et al., 2021).

En el caso de la educación superior, existen fuertes desigualdades según la condición socioeconómica de los estudiantes (Díaz, et al., 2021). En la comentada situación de pandemia, $\mathrm{y}$ enseñanza online, una de las mayores preocupaciones que expresa la muestra estudiada de docentes universitarios que dictan la materia de IO, es cómo intentar superara la denominada "brecha 
digital y el acceso limitado a las tecnologías". Los docentes expresan que muchos estudiantes tienen dificultades para acceder a computadoras o no tienen conectividad a su disposición, para poder trabajar online los diferentes contenidos educativos. En este contexto, según Ordorika (2020) en los próximos meses y años es muy probable que se den cambios relevantes en las formas tradicionales de trabajo académico y en la organización de las IES. En la misma línea, Moser, et al., (2021) expone que será necesario analizar con cuidado los problemas y alcances que la educación a distancia ha evidenciado durante la crisis actual, para superar las deficiencias pedagógicas y la agudización de la exclusión y la desigualdad social y de género. Según López, et al., (2021) ya se habla del establecimiento de nuevos modelos de enseñanza virtual y de sistemas híbridos (presenciales y a distancia) para la docencia. Así pues, el $86.66 \%$ de los docentes universitarios objeto de estudio que dictan la materia de IO, están de acuerdo con utilizar los recursos y aplicaciones oficiales que ofrecen las IES (correo electrónico institucional, plataformas online, aulas virtuales, entre otros), y así evitar toda la posible problemática relacionada con el uso de documentos, imágenes y protección de datos.

Aunque hay miles de publicaciones sobre los beneficios del uso de TIC en la educación y, más concretamente, de las tecnologías de aprendizaje y conocimiento (TAC), lo cierto es que la calidad y la motivación en la educación en línea es difícil de lograr si la brecha digital sigue aquí y los educadores carecen de recursos y formación (Olivares et al., 2021). Esta situación ha afectado en mayor o menor medida a diferentes zonas de Ecuador (Usca-Veloz, et al., 2021). En este caso, esta investigación ha analizado la experiencia de los docentes universitarios que dictan la materia de IO en las provincias ecuatorianas de Bolívar, Chimborazo, Manabí, y Tungurahua, quienes sostienen que impartir clases online debe sustentarse en una cultura de pedagogía virtual, puesto que consideran que no solo es un cambio de escenario del aula al hogar. El 93.33\% de los académicos desde su experiencia expresan que desde el inicio de las actividades académicas online se ha evidenciado diversas particularidades que coexisten entre los estudiantes universitarios, supuestos "nativos digitales" a la hora de demostrar sus habilidades, estas situaciones son resultado de que varias familias no tienen suficientes ordenadores para poder teletrabajar y estudiar afectando significativamente al grupo estudiantil más desfavorecido; los estudiantes desconectados.

El análisis de la situación actual del estado de la cuestión; nos lleva a comprender que los docentes universitarios que dictan la materia de IO propenden por lograr aprendizajes en sus estudiosos, desarrollar distintas capacidades que formen de manera integral a la persona su pensamiento, la acción, el ser, la comunicación, la relación con el contexto y la posibilidad de aplicación de sus aprendizajes en el mundo laboral además de aportar a su desarrollo social, son otras de las labores del docente en cualquiera de las etapas de la educación.

El docente en el desarrollo de sus actividades se ve abocado a una sucesión de procesos que se convierten en carga laboral física, emocional, mental, familiar entre otras, todo lo anterior en situación de normalidad académica, el estilo de vida de los maestros universitarios se ve sin lugar a duda afectado por causas internas y externas diferentes del entorno laboral lo que forja necesarios abordajes en su calidad de vida a partir de múltiples perspectivas, con amplitud de criterio y con la concepción sistémica y compleja de la realidad, asumiendo los escenarios de certeza, sin eludir el océano de incertidumbres en los que se hallan los involucrados, pretendiendo configurar la red de interrelaciones de sus múltiples elementos y dimensiones (Moser, et al., 2021). Así lo evidencia el presente estudio, puesto que el $93.33 \%$ de la 
muestra de académicos universitarios que dictan la materia de IO desde su experiencia expresan que el reto para la IES ante la COVID-19 es conducir esta crisis a un aprendizaje significativo y constructivo en vez del debilitamiento del estudiante, con el fin de desarrollar diversas alternativas para establecer un marco flexible que condescendiese a los docentes adoptar las medidas que meditasen más adecuadas para el desarrollo de las actividades de aprendizaje y evaluación, teniendo en cuenta las tipologías específicas la asignatura y las directrices dadas por los entes rectores de educación superior.

Numerosos modelos pedagógicos, fundamentados en el uso de internet, han sido analizados y puestos en práctica. Autores como Digión y Álvarez (2021) evidencian que este nuevo planteamiento pedagógico permite entornos de aprendizajes adaptativos y autorregulados, pues propician la aparición de un nuevo agente educador computacional con retroalimentación continua y rápida del logro académico alcanzado. En esta línea, Awidi, et al., (2019); Saura (2020); Issa, et al., (2021) han comprobado que plataformas virtuales, confeccionadas a modo de red social, permiten una mejora significativa de la actitud del alumnado y facilitan el uso de espacios de debate, sistemas de mensajería directa alumno-docente, y las herramientas necesarias para el planteamiento de actividades y su envío, una vez realizadas, telemáticamente. Bajo este contexto, y tomando en consideración las múltiples plataformas existentes, los docentes universitarios de IO exponen que han utilizado, en mayor medida se encuentran las aulas virtuales Moodle y el correo electrónico corporativo, este último utilizado por un $46.66 \%$ de los docentes. El 40.00\% de los docentes utilizan la herramienta Teams. Otra parte de los docentes ha optado por trabajar en la creación de blogs personales y páginas web como: Google Pages, Google Classroom, y canales de YouTube. El 50\% utiliza herramientas combinadas con la App Zoom para videoconferencias. Por ultimo un $16.66 \%$ usan redes sociales como WhatsApp o Instagram para comunicarse con los estudiantes.

En estas circunstancias, la actual sociedad del conocimiento obliga a plantearse nuevas ideas y concepciones sobre el aprendizaje y las estrategias pedagógicas para alcanzarlo, de manera especial en el desarrollo de competencias digitales en los docentes y estudiantes en formación, quienes luego influirán en las nuevas generaciones de alumnos (Portillo, et al., 2020). Al respecto, los docentes universitarios del grupo de trabajo opinaron que un aspecto muy relevante es la evaluación y monitoreo de los aprendizajes, así como la retroalimentación, es trascendental para conocer el progreso de las y los estudiantes y tomar las acciones pedagógicas pertinentes a fin de mejorarlo, plantean además un análisis y proponen la necesidad de un proceso de formación del docente en TIC; ya que como señalan Buitrago, et al., (2020) si los docentes universitarios, y profesionales en formación (estudiantes universitarios), utilizan y manejan las TIC también lo harán durante el desempeño de su profesión. Ante esta situación, el grupo de trabajo opinó que, parte de la solución sería modificar los planes curriculares de forma que los estudiantes universitarios puedan alcanzar no solamente competencias digitales sino además mediáticas, desarrollando enérgicamente un desempeño crítico en la sociedad digital y más exitoso en el mundo laboral. Por ello, los currículos deberán ser de calidad, coherentes con los requerimientos actuales y revisados anualmente.

\section{CONCLUSIONES}

A partir de los resultados de este estudio y las evidencias recolectadas de las reflexiones de la enseñanza virtual de la IO durante la COVID-19, partiendo de análisis desde la práctica docente universitaria, se puede decir que la pandemia de la COVID 19 afectó significativamente a la 
sociedad en su conjunto y especialmente a los procesos propios de la educación superior, y reveló profundas limitaciones de carácter organizativo, administrativo y pedagógico en la implementación de entornos virtuales de aprendizaje y en demostró, la poca capacidad para responder con eficiencia a las demandas o exigencias de situaciones fortuitas. Debido a ello, buscar soluciones se convirtió en algo ineludible, en este entorno se tomó en cuenta los recursos disponibles (presentaciones de PowerPoint, recursos complementarios o simuladores), al igual que los espacios habilitados para la comunicación y la evaluación continua del proceso de aprendizaje (correo electrónico institucional, plataformas online, aulas virtuales, entre otros). Por ello, y tras un escenario pandémico, la docencia online se cristianizó en la única solución posible e incumbía ponerse en marcha lo antes posible.

La modalidad de educación online, virtual, a distancia y remota de emergencia, constituye un recurso de esencial importancia para concretar la formación profesional en las Instituciones de Educación Superior (IES) que albergan distintos campos del conocimiento. Por lo tanto, su implementación no debe limitarse a la imposibilidad de recibir clases presenciales, sino más bien, fortalecerse paralelamente considerando sus ventajas frente a las exigencias de una sociedad que cambia y se transforma permanentemente. A estos se suman la necesidad de un establecimiento de nuevos modelos de enseñanza virtual y de sistemas híbridos (presenciales y a distancia) para la docencia. A más de promover procesos de democratización de las IES, tanto en el nombramiento de autoridades como en la toma de decisiones. Para ello, será imperioso tomar iniciativas para el mejoramiento de las condiciones laborales y salariales de los docentes contratados considerando que estos representan el $60.00 \%$ de la muestra de estudio.
Futuras líneas de investigación podrían ampliar el alcance del estudio y abordar, desde la perspectiva del docente universitario, el modelo de enseñanza online que han implementado durante la suspensión de las clases presenciales e identificar las dificultades que han enfrentado unos y las buenas prácticas de otros. Este artículo busca enérgicamente presentar mediante evidencias empíricas un análisis con luces y sombras sobre la enseñanza virtual impartida durante la COVID-19, a partir de una disertación desde la práctica docente universitaria, que sirva a las IES para afrontar los retos que sigue deparando la COVID-19.

\section{REFERENCIAS}

Awidi, I. T., Paynter, M., \& Vujosevic, T. (2019). Facebook group in the learning design of a higher education course: An analysis of factors influencing positive learning experience for students. Computers and Education, 129(November 2018), 106-121. https://doi. org/10.1016/j.compedu.2018.10.018

Buitrago, A. R. A., Gutiérrez, O. Á. de J., \& Romero, R. N. (2020). Inmigrantes digitales vs. nativos digitales en instituciones educativas públicas Venezolanas: Aforismos sobre una realidad poliédrica controvertida. Revista Saperes Universitas, 4(1), 6-38. doi:http://dx.doi. org/10.16967/rpe.v4n2a2.

Caicedo, S. N., \& Ruiz, C. R. D. (2021). Estándares mínimos de calidad de la educación superior en Ecuador durante la pandemia por COVID-19, como medio de protección del derecho a la educación superior. TSAFIQUI | Revista Científica en Ciencias Sociales, 16(1), 7-17. https://doi.org/https://doi.org/10.29019/ tsafiqui.v12i16.877

Castillo, S. L. A., \& Cabrera, C. S. M. (2021). La educación virtual implementada por la pandemia de la COVID-19 y el derecho a la educación superior. Crítica y Derecho: Revista Jurídica, 2(3), 44-56. https://doi.org/https://doi. org/10.29166/cyd.v2i3.3188 
Coronel, T. (2021). De las pizarras a las pantallas , un reto docente en Ecuador. Revista de Experiencias Pedagógicas MAMAKUNA, 1(16), 48-55.

Díaz, V. J., Ruiz, R. A., \& Egüez, C. C. (2021). Impacto de las TIC: desafíos y oportunidades de la Educación Superior frente al COVID-19. Uisrael Revista Científica, 8(2), 113-134. https://doi.org/https://doi.org/10.35290/rcui. v8n2.2021.448

Digión, L. B., \& Álvarez, M. M. (2021). Experiencia de enseñanza- aprendizaje con aula virtual en el acompañamiento pedagógico debido al Covid-19. Apertura. Revista de Innovación Educativa, 13(1), 20-35. https://doi.org/http:// doi.org/10.32870/Ap.v13n1.1957

García, M. M. A., Condo, L. C. de L., Quiñonez, C. P., Alvarado, M. J. R., \& Salas, A. F. G. de G. (2021). Uso de TIC en la prevención del dengue asociado al proceso enseñanza-aprendizaje de enfermería clínica, Universidad de Guayaquil , Ecuador 2020. Boletín de Malariología y Salud Ambiental, 1(2), 175-182.

Gorozidis, G. S., Tzioumakis, Y. S., Krommidas, C., \& Papaioannou, A. G. (2020). Facebook group PETCoN (Physical Education Teacher Collaborative Network). An innovative approach to PE teacher in-service training: A self-determination theory perspective. Teaching and Teacher Education, 96, 103184. https://doi. org/10.1016/j.tate.2020.103184

Issa, T., Alqahtani, S. G. B., Al-Oqily, I., Goktalay, S. B., Köse, U., Issa, T., Abu Salih, B., \& Almufaraj, W. K. (2021). Use of social networking in the Middle East: student perspectives in higher education. Heliyon, 7(4). https://doi. org/10.1016/j.heliyon.2021.e06676

Kang, D., \& Jae, M. (2017). Telematics and Informatics Competitive prospects of graduate program on the integration of ICT superiority, higher education, and international aid. Telematics and Informatics, 34(8), 1625-1637. https://doi.org/10.1016/j.tele.2017.07.009

López, M., Herrera, M., \& Apolo, D. (2021). Educación de calidad y pandemia: retos, experiencias y propuestas desde estudiantes en formación docente de Ecuador. Linguagem e Tecnologia, 14(2), 1-12. https://doi. org/10.35699/1983-3652.2021.33991

López, Y., Ferrer, H. ilda, Caballero, Y., Guerra, L., Junco, R., Benítez, I., Rodriguez, A., \& Madera, J. (2020). Aplicación de la investigación de operaciones a la distribución de recursos relacionados con la COVID-19. Retos de La Dirección, 14(2), 86-105.

Monterubbianesi, P. D., Rojas, L. M., \& Dabús, C. D. (2021). Educación y salud : evidencia de efectos umbral en el crecimiento económico. Lecturas de Economía, 94(1), 195-231. https://doi.org/ https://doi.org/10.17533/udea.le.n94a342459

Moser, K. M., Wei, T., \& Brenner, D. (2021). Remote teaching during COVID-19: Implications from a national survey of language educators. System, 97, 102431. https://doi.org/10.1016/j. system.2020.102431

Olivares, S. T., Vázquez, A. M., \& Toledano, R. M. (2021). La Docencia Virtual o e-Learning como Solución a la Enseñanza de la Física y Química de los Futuros Maestros en tiempos de COVID-19. Revista Española de Educación Comparada, 38(extra), 190-210. https://doi. org/10.5944/reec.38.2021.28853

Ordorika, I. (2020). Pandemia y educación superior. Revista de La Educación Superior, 49(1), 1-8. https://doi.org/https://doi.org/10.36857/ resu.2020.194.1120

Portillo, P. S. A., Castellanos, P. L. I., Reynoso, G. Ó. U., \& Gavotto, N. O. I. (2020). Enseñanza remota de emergencia ante la pandemia Covid-19 en Educación Media Superior yEducación Superior. Propósitos y Representaciones, 8(3), e589. https:// doi.org/10.20511/pyr2020.v8nspe3.589

Salas, D. M. (2020). Convergencia entre Nativos Digitales e Inmigrantes Digitales. Sinergias Educativas, 1(5), 1-12.

Salazar, F. (2021). Modelo matemático de optimización permite asignar pacientes a terapeutas para intervención en crisis durante emergencia COVID-19. Revista ASOiMAT EPN, $\operatorname{VII}(1), 16-22$. 
Saura, G. (2020). Filantrocapitalismo digital en educación: Covid-19, UNESCO, Google, Facebook. Teknokultura. Revista de Cultura Digital y Movimientos Sociales, 17(2), 159-168.

Usca-Veloz, R. B., Muyulema-Allaica, J. C., \& Velasteguí-Bósquez, G. A. (2021). Integrando electricidad, ondas y calor en la enseñanza de la física antes y durante el confinamiento por COVID-19. Revista Conciencia Digital, 4(1.2), 405-421. https://doi.org/https://doi. org/10.33262/concienciadigital.v4i1.2.1607

Vera, F. (2021). Impacto de las plataformas de videoconferencia en la educación superior en tiempos de COVID-19. Revista Electrónica Transformar, 2(1), 41-57. 